pressure at the melting point, the affinity of the deposited films for the residual gases in the vacuum system, and the depression of the superconducting transition temperature by the gettered residual gases in the films. Tantalum is a metal in which similar difficulties are encountered; Marchand and Venema ${ }^{4}$ have shown that tantalum films with bulk metal superconducting transition properties can be prepared in vacua of the order of $1 \times 10^{-10}$ torr by sublimation from a heated tantalum spiral at a deposition-rate of $250 \AA$./min. We have estimated that in order to produce pure niobium films by vacuum sublimation a vacuum of at least $1 \times 10^{-11}$ torr would be required.

We are not aware that superconducting films of niobium have been reported previously.

By the use of a vacuum-arc deposition technique ${ }^{5,6}$ developed in this laboratory, we have been able to produce superconducting niobium films with transition temperatures greater than $4 \cdot 2^{\circ} \mathrm{K}$., which is the present upper limit of measurement in our cryostat. Cook, Zemansky, and Boorse ${ }^{7}$ obtained a value of $8 \cdot 65^{\circ} \mathrm{K}$. for the transition temperature of bulk niobium. Our niobium films were deposited on to $25 \mathrm{~mm} . \times 10 \mathrm{~mm}$. glass substrates in vacua of $5-$ $8 \times 10^{-6}$ torr, during deposition the centre of each substrate was placed at a distance of $3 \mathrm{~mm}$. from the point of contact of the two are electrodes. The electrical resistance of the films range from $0.93 \mathrm{ohms}$ per square to $6 \cdot 1 \mathrm{ohms}$ per square at $300^{\circ} \mathrm{K}$.

In view of the present interest in this type of super. conductor, we believe that these preliminary results may be of some use to other workers in this field. No doubt there are other areas where the vacuumarc deposition technique can be used to obtain similar advantages over contemporary methods. This work was performed under contract with the United States Atomic Fnergy Commission.

M. S. P. LUCAS

D. T. MEYER

Superconducting Circuits Laboratory,

Electrical Engineering Department, Duke University,

Durham, North Carolina.

${ }^{1}$ Holland, L., Nature, 178, 328 (1956).

${ }^{2}$ Hiesinger, L., German Patent No. 915765 (July 29, 1954).

${ }^{3}$ Vodar, B., Minn, S., and Offret, S., J. Phys, Rad., 16, 811 (1955).

- Marchand, J. F., and Venema, A., Philips Res. Rep., 14, 427 (1959).

${ }^{5}$ Lucas, M. S. P., Owen, H. A., Stewart, W. C., and Vail, C. R., Rev. Sci. Instr., 32, 203 (1961).

- Lucas, M. S. P., Vail, C. R., Stewart, W. C., Owen, H. A., Vacuum Symposium Transactions, Eighth Nat. Symp. (Pergamon Press) (in the press).

' Cook, D. B., Zemansky, M. W., and Boorse, H. A., Phys. Rev., 80, $737(1950)$.

\section{Search for Light Amplification in a Mixture of Mercury Vapour and Hydrogen}

F. A. Butayeva and V. A. Fabrikant have recently reported the discovery of a negative temperature in an exeited gaseous mixture of mercury vapour and hydrogen between the levels $7^{3} S_{1}$ and $6^{3} P_{0,1,2}$ of mercury ${ }^{1}$. They maintain that the populations of the $6^{3} P$ levels were reduced below that of the $7^{3} S_{1}$ level by the well-known quenching action of hydrogen. The populations were investigated by measuring the transmission coefficient of the gas mixture at the wave-lengths $5461 \AA ., 4358 \AA$. and $4047 \AA$., corresponding to the transitions $7^{3} S_{1}-6^{3} P_{2}, 7^{3} S_{1}-6^{3} P_{1}$ and $7^{3} S_{1}-6^{3} P_{0}$, the mixture being excited by a d.c. discharge. With a discharge tube of length $36 \mathrm{~cm}$. amplification of about 10 per cent was reported in the $\lambda 5461 \AA$. and $\lambda 4358 \AA$. lines for a discharge current of $70 \mathrm{~m}$.amp. with $0.3 \mathrm{~mm}$. mereury hydrogen pressure and saturated mercury vapour at room temperature.

In similar experiments with this mixture, under conditions as nearly as possible identical to those used by Butayeva and Fabrikant, and with a wide variation of hydrogen pressure, we have observed no amplification.

In our apparatus light from a low-pressure mercury lamp was chopped at a low frequency and then passed through the discharge. The line to be investigated was selected with a monochromator, and the light fell on a photomultiplier. A second photomultiplier was used to monitor the light output from the source lamp and the a.c. output from the two photomultipliers was electronically balanced and fed to a phase-sensitive detector. With this arrangement 1 per cent amplification could be detected with ease. The discharge tube was baked at $400^{\circ} \mathrm{C}$. for at least $8 \mathrm{hr}$. before attempting measurements and the final pressure achieved before admitting the gases was 10-8 mm. mercury. The hydrogen was admitted by diffusion through hot palladium and the mercury was pre-distilled under vacuum.

With d.c. excitation of the discharge the current was varied continuously from 0 to $150 \mathrm{~m}$.amp. with hydrogen pressures ranging from 0.2 to $0.6 \mathrm{~mm}$. mercury. No absorption or amplification was observed in any of the three lines. At hydrogen pressures below $0 \cdot 2 \mathrm{~mm}$. mercury, difficulty was experienced in maintaining a d.c. discharge, but it was possible to run a radio-frequency electrodeless discharge at about $10 \mathrm{Mc} / \mathrm{s}$. Investigations were made with the latter form of excitation over the range of hydrogen pressures of $0-0.6 \mathrm{~mm}$. mercury. Again no effect was obtained in the $\lambda 4358 \AA$. line, and a small absorption was observed in the $\lambda 4047 \AA$. line. Strong absorption was, however, observed at the lowest pressures in the $\lambda 5461 \AA$. line, being typically about 50 per cent at zero hydrogen pressure, depending on the radiofrequency power used; in this case the absorption fell to, and remained at, zero as the hydrogen pressure was increased up to and beyond about $0.15 \mathrm{~mm}$. mercury. In all cases the mercury vapour was in equilibrium with liquid mercury at a temperature of $20 \pm 2^{\circ} \mathrm{C}$.

It is difficult to form a convincing theoretical discussion of the conditions in the discharge since the quenching process is not fully understood. However, the experimentally determined cross-section of $8.6 \times 10^{-18} \mathrm{~cm} .^{2}$ (ref. 2) for quenching of atoms in the $6^{3} P_{1}$ state is not large enough for this process alone to create a negative temperature under these conditions, even supposing that the life-time of atoms in the $7^{3} S_{1}$ state is not affected by the hydrogen. This lends support to our experimental conclusion.

The authors are grateful to Dr. B. A. Lengyel for supplying them with a translation of the Russian report.

J. H. SANDERS

M. J. TAYLOR

C. E. WEBB

Clarendon Laboratory, Oxford. 1 Butayeva, F. A., and Fabrikant, V. A., Research in Experimental
and Theoretical Physics, Memorial Vol. in Honour of G. S. Landsberg (U.S.S.R. Academy of Science Press, Moscow, 1959).

${ }^{2}$ Pringsheim, P., Fluorescence and Phosphorescence, 111 (Interscience 